\title{
Análise do perfil do paciente suicida: revisão integrativa
}

\author{
Analysis of the suicide patient profile: integrative review \\ Analisis del perfil del paciente suicida: revisión integrativa
}

Raíssa Gonçalves Pereira ${ }^{1 *}$, Bentinelis Braga da Conceição ${ }^{1}$, Yohanna Larissa Soares Damasceno ${ }^{1}$, Gêzana Rita Cunha Oliveira ${ }^{1}$, Rhanyele de Moura Cardoso ${ }^{1}$, Josélia Martins de Medeiros $^{1}$, Naiane Almeida da Silva ${ }^{1}$, Kadja Fernanda Tinoco ${ }^{1}$, Vaniele dos Santos da Silva de Oliveira $^{1}$, Antônia Ângela da Conceição Salazar ${ }^{1}$, Dayane Vitória da Silva Santos ${ }^{1}$, Nataly Camila Gomes de Arrais Figueiredo ${ }^{1}$, Kellen Rayane de Oliveira Castro ${ }^{1}$, Camilla Lohanny Azevedo Viana ${ }^{1}$, Elizabeth Christina Silva Fenandes².

\section{RESUMO}

Objetivo: Identificar por meio da literatura qual o perfil do paciente suicida. Metodos: O presente estudo tratase de uma revisão integrativa da literatura, elaborada através da consulta de descritores nas bases de dados PubMed; BVS (Biblioteca Virtual da Saúde), e EBSCO no período de novembro 2018 a janeiro de 2019. Os critérios de inclusão foram estudos disponíveis em sua totalidade, publicados nos últimos cinco anos (2014 a 2019), e estudos publicados nos idiomas Português, Espanhol e Inglês. Foram excluídas outras formas de publicação que não fossem artigos científicos completos. Resultados: A maioria das vítimas de tentativa de suicídio são jovens do gênero masculino. Quanto ao perfil emocional da pessoa suicida segundo os estudos, foi relacionado um perfil de pessoas com depressão, ansiedade, melancolismo, com baixo autoestima, sentindo-se desamparados, e com desesperança em relação a vida. Considerações finais: Conclui-se que os jovens do gênero masculino são mais propensos a tentarem suicídio, e que os profissionais de saúde têm importante papel na prevenção do suicídio, pois eles garantem uma assistência de qualidade e de acordo com as necessidades físicas e psicoemocionais dos pacientes.

Palavras-chave: Suicídio, Perfil, Saúde mental.

\begin{abstract}
Objective: To identify through the literature the profile of the suicidal patient. Methods: The present study is an integrative review of the literature, elaborated through the query of descriptors in PubMed databases; VHL (Virtual Health Library), and EBSCO from November 2018 to January 2019. The inclusion criteria were studies available in their entirety, published in the last five years (2014 to 2019), and studies published in Portuguese, Spanish and Portuguese. English. Other forms of publication other than full scientific articles were excluded. Results: Most suicide attempt victims are young males. As for the suicidal person's emotional profile according to the studies, a profile of people with depression, anxiety, melancholy, low self-esteem, feeling helpless, and with hopelessness about life was related. Conclusions: It is concluded that young males are more likely to attempt suicide, and that health professionals play an important role in suicide prevention, since they guarantee quality care and in accordance with the physical and psycho-emotional needs of the patients.
\end{abstract}

Key words: Suicide, Profile, Mental health.

${ }^{1}$ Centro Universitário de Ciências e Tecnologia do Maranhão (UniFacema), Caxias-MA.

*E-mail: raissa.goncalvez@hotmail.com

${ }^{2}$ Universidade Estadual do Piauí (UESPI), Teresina-PI.

SUBMETIDO EM: $\mathbf{3 / 2 0 1 9}$ 


\section{RESUMEN}

Objetivo: Identificar por medio de la literatura cuál es el perfil del paciente suicida. Metodos: El presente estudio se trata de una revisión integrativa de la literatura, elaborada a través de la consulta de descriptores en las bases de datos PubMed; BVS (Biblioteca Virtual en Salud), y EBSCO en el período de enero de 2018 a janiero de 2019. Los criterios de inclusión fueron los estudios disponibles en su totalidad, publicados en los últimos cinco años (2014-2019), y estudios publicados en portugués, español y Inglés. Se excluyeron otras formas de publicación que no fueran artículos científicos completos. Resultados: La mayoría de las víctimas de intento de suicidio son jóvenes del género masculino. En cuanto al perfil emocional de la persona suicida según los estudios, se relacionó un perfil de personas con depresión, ansiedad, melancolismo, con bajo autoestima, sintiéndose desamparados, y con desesperanza en relación a la vida. Consideraciones finales: Se concluye que los jóvenes del género masculino son más propensos a intentar suicidios, y que los profesionales de salud desempeñan un papel importante en la prevención del suicidio, ya que garantizan una asistencia de calidad y de acuerdo con las necesidades físicas y psicoemocionales pacientes.

Palabras clave: Suicidio, Perfil, Salud mental.

\section{INTRODUÇÃO}

O suicídio é a morte resultada de um ato executado pelo próprio indivíduo, consciente do desfecho final. O sujeito suicida vive uma falha emocional, vivida como excesso ou perda de sentimentos, que podem ser propensos ao ato suicida, que é uma tentativa de pôr fim na condição desesperadora enfrentada pelo paciente (FREITAS G, 2015).

Os fatores de riscos para o ato suicida incluem transtornos mentais, sócio-demográficos, psicológicos e condições clínicas incapacitantes. Dentre os transtornos mentais mais frequentes encontram-se os de humor, personalidade, esquizofrenia e aqueles decorrente do uso de substâncias psicoativas (DUTRA EMS, 2008).

Partindo da definição de Botega NJ (2017), em que considera o suicídio um problema "oculto" na sociedade, este é evidenciado por cerca de um milhão de pessoas, e corresponde a aproximadamente uma morte a cada 40 segundos por ano, sendo que em 2015 o numero de suicídios no Brasil foram 11.178, um ano depois em 2016 foram 11.433 mortes por suicídio, e a tendência é um aumento progressivo desses números, em média 2\% por ano. Diante do exposto a presente pesquisa indaga: Quais evidencias existentes na literatura sobre o perfil do paciente suicida?

Considerando a importância de discutir e estruturar trabalhos sobre suicídio, a presente pesquisa justificouse com o intuito de contribuir para conhecimentos na área com a tentativa de avaliar os principais fatores de risco para suicídio, visando contribuir também para a construção do conhecimento no campo da assistencial da saúde, tendo em vista, que o cliente que comete tentativa suicida necessita de cuidados na área da saúde mental.

Assim, este estudo tem como objetivo geral: Identificar por meio da literatura qual o perfil do paciente suicida. E como objetivos específicos: Identificar através da literatura o período da vida humana com maior incidência de morte por suicídio; pesquisar na literatura os principais fatores que podem contribuir para o suicídio; analisar através da literatura qual o estado emocional mais recorrente das pessoas que são vítimas de suicídio.

\section{MÉTODOS}

O presente estudo trata-se de uma pesquisa bibliográfica do tipo revisão integrativa da literatura. Este procedimento foi escolhido por possibilitar a síntese e análise do conhecimento científico já produzido sobre o tema "ANALISE DO PERFIL DO PACIENTE SUICIDA: revisão integrativa", que determinou a construção da estratégia PICo, que representa um acrônimo para Paciente (P), Intervenção (I) e Contexto (Co), na qual foi utilizada para a geração da questão norteadora desta revisão integrativa da literatura: Quais evidencias existentes na literatura sobre o perfil do paciente suicida? 
Para a localização dos estudos relevantes, que respondessem à pergunta de pesquisa, utilizou-se de descritores indexados nos idiomas português, inglês e espanhol. Os descritores foram obtidos a partir do Medical Subject Headings (MESH), dos Descritores em Ciências da Saúde (DeCS) e dos títulos EBSCO, como mostra o quadro 1. Consultou-se por meio dos descritores as bases de dados PubMed da National Library of Medicine; BVS (Biblioteca Virtual da Saúde), coordenada pela BIREME e composta de bases de dados bibliográficas produzidas pela Rede BVS, como LILACS, além da base de dados Medline e outros tipos de fontes de informação; e EBSCOhost.

Quadro 1 - Elementos da estratégia PICo e descritores utilizados - Caxias, MA, Brasil, 2019.

\begin{tabular}{|l|l|l|}
\hline ELEMENTOS & MESH & DECS \\
\hline $\mathrm{P}=$ Paciente & Suicidal Ideation. & Ideação Suicida; Ideación Suicida; Suicidal Ideation. \\
\hline $\mathrm{I}=$ intervenção & Sickness Impact Profile & $\begin{array}{l}\text { Perfil de Impacto da Doença; Perfil de Impacto de } \\
\text { Enfermedad; Sickness Impact Profile. }\end{array}$ \\
\hline Co = contexto & Emotional. & Emocional; Emocional; Emotional. \\
\hline
\end{tabular}

Fonte: pesquisa direta, 2019.

Os descritores utilizados nessa revisão integrativa foram combinados nos bancos de dados das bases escolhidas, resultando em estratégias sistematizadas de acordo com a proposta da pergunta norteadora da pesquisa. A pesquisa ocorreu entre os meses de novembro 2018 a janeiro de 2019.

Como critérios de inclusão foram utilizados de estudos disponível em sua totalidade, publicados nos últimos cinco anos (2014 a 2019), e somente estudos nos idiomas Português, Espanhol e Inglês. Foram excluídos da busca inicial capítulos de livros, resumos, textos incompletos, teses, dissertações, monografias, relatos técnicos e outras formas de publicação que não fossem artigos científicos completos.

Para organização e análise dos estudos escolhidos para comporem os resultados dessa revisão integrativa primeiramente foi elaborada uma pré-seleção de artigos conforme os critérios de inclusão e exclusão definidos na metodologia. Na base de dados BVS através busca geral com a combinação de descritores foi resultante em treze artigos, estes foram filtrados na base de dados conforme os critérios de inclusão e exclusão, descartando-se os artigos que não estavam disponíveis em sua totalidade, fora do recorte temporal de 2014 a 2019, e publicados em idiomas que não fosse português, inglês ou espanhol, limitando os estudos antes selecionados na busca geral para apenas sete. Estes ainda foram filtrados para exclusão de artigos duplicados durante a busca e de artigos que fugissem a questão norteadora do estudo, sendo visto que três artigos eram condizentes com os critérios e os objetivos dessa pesquisa.

Para a análise e seleção dos estudos, primeiramente foram buscados artigos através da combinação de descritores. Na base de dados BVS, foram encontrados treze (13) estudos na busca inicial, logo após os estudos foram filtrados de acordo com os critérios de inclusão e exclusão resultando em sete (07) artigos, estes ainda foram analisados para verificação de artigos duplicados ou que não correspondessem a temática, assim apenas três (03) deles foram condizentes com o objetivo desse trabalho.

$\mathrm{Na}$ base de dados PUBMED, conforme a estratégia de busca com a combinação de descritores, foram obtidos dois (02) estudos na busca total. Tal como feito na base anterior, os estudos passaram pela filtragem de acordo com os critérios de inclusão e exclusão estabelecidos, continuando com o total de dois (02) estudos, que condisseram com a temática proposta.

$\mathrm{Na}$ EBSCO foram obtidos onze (11) estudos como busca geral, sendo que limitando a busca pelos últimos cinco anos, obteve-se cinco (05) estudos, sendo selecionado três (03) que condisseram com o tema.

A Figura 1 demonstra o esquema de análise e seleção dos estudos das três bases de dados utilizadas, onde é possível analisar mais claramente o fluxograma do processo de seleção dos artigos que compuseram os resultados dessa pesquisa. 
Figura 1- Fluxograma do processo de seleção dos estudos para a revisão integrativa, Caxias- MA, 2019.

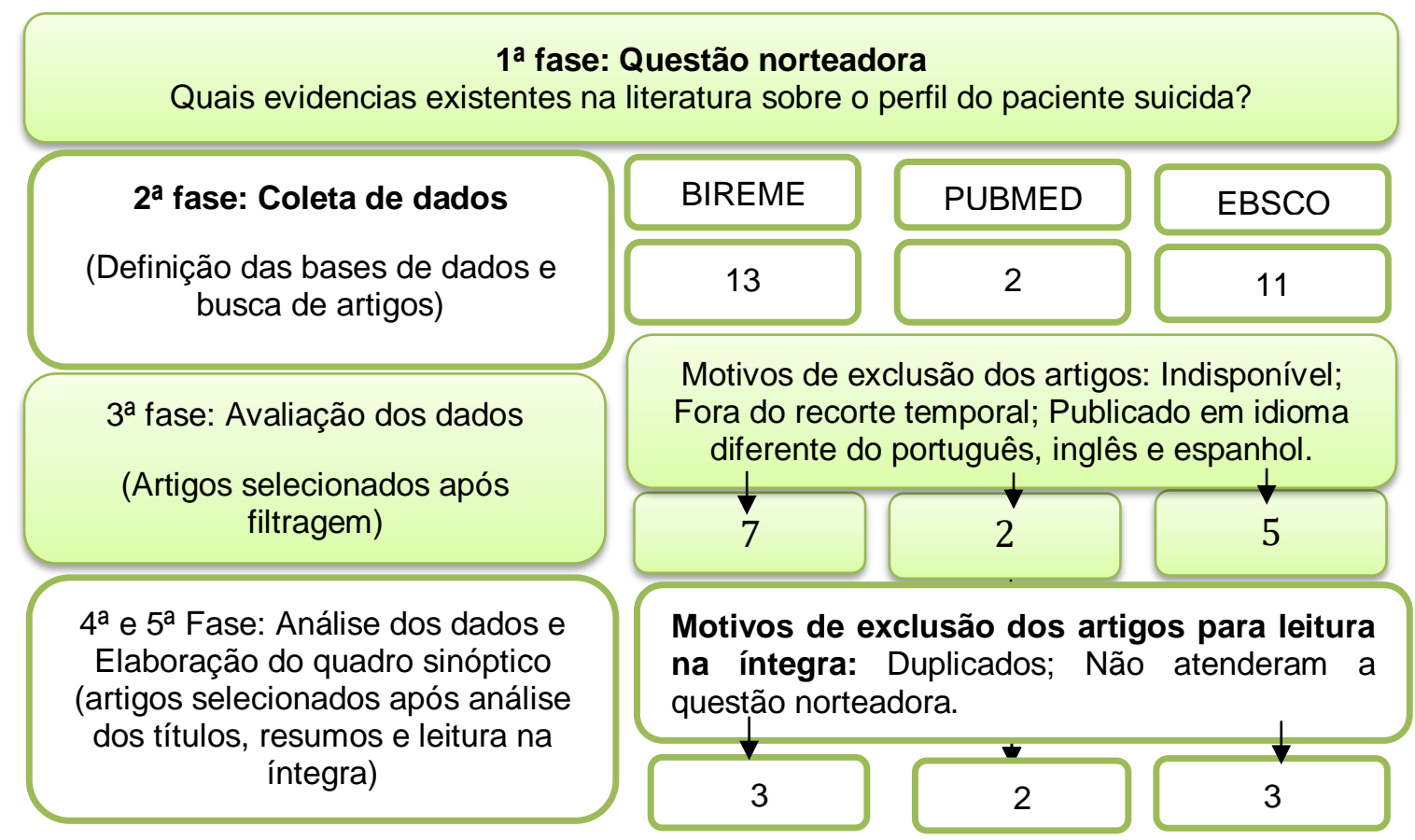

Fonte: BIREME, PUBMED e EBSCO, 2019.

\section{RESULTADOS}

Essa pesquisa resultou em oito artigos oriundos das bases de dados online BRIREME/BVS, PUBMED e EBSCO, tendo prevalência BIREME e EBSCO, havendo predominância de artigos qualitativos, sendo a maioria concentrados no ano de 2017, quanto a procedência as análises foram em sua maioria provenientes do Brasil (Tabela 2).

Tabela 2 - Caracterização das produções analisadas, Bireme-BVS, PubMed e EBSCO. Caxias, Maranhão, Brasil, 2019.

\begin{tabular}{lccc}
\hline Variáveis & N & $\%$ \\
\hline Quantitativo & Abordagem de estudo & 1 & 12,5 \\
Qualitativo & Fonte online & 7 & 87,5 \\
& & 3 & 37,5 \\
Bireme/BVS & & 2 & 25,0 \\
PubMed & Distribuição temporal & 3 & 37,5 \\
EBSCO & & 2 & \\
& & 1 & 25,0 \\
2014 & & 1 & 12,5 \\
2015 & & 3 & 12,5 \\
2016 & & 1 & 34,5 \\
2017 & & & 12,5 \\
2018 & Procedência & 7 & 87,5 \\
Brasil & & 1 & 12,5 \\
México & & & \\
\hline
\end{tabular}

Fonte: BIREME, PUBMED e EBSCO, 2019. 
O quadro 2 evidencia que as publicações incluídas tinham procedência da realização do estudo em sua maioria no Brasil, delineamento da pesquisa predominantemente sendo estudo descritivo do tipo exploratório, com a maioria pertencendo ao nível 5 de evidência e grau de recomendação B. O nível 5 cita serie de casos e o grau de recomendação $B$ representa resultados não conclusivos dos estudos.

Quadro 2 - Distribuições das publicações incluídas segundo o título, ano de publicação, procedência da realização do estudo, delineamento da pesquisa, nível de evidência e grau de recomendação. Caxias, Maranhão, Brasil, 2019.

\begin{tabular}{|c|c|c|c|c|c|c|}
\hline $\begin{array}{l}\text { № de } \\
\text { ordem }\end{array}$ & Título & $\begin{array}{l}\text { Base/Ano de } \\
\text { publicação }\end{array}$ & Procedência & $\begin{array}{c}\text { Delineamento } \\
\text { da pesquisa }\end{array}$ & $\begin{array}{l}\text { Nível de } \\
\text { evidência }\end{array}$ & $\begin{array}{c}\text { Grau de } \\
\text { recomendação }\end{array}$ \\
\hline A1 & $\begin{array}{l}\text { Perfil de pacientes } \\
\text { atendidos por } \\
\text { tentativa de suicídio } \\
\text { em um centro de } \\
\text { assistência } \\
\text { toxicológica. }\end{array}$ & $\begin{array}{l}\text { BIREME/ } \\
\text { BVS } 2015\end{array}$ & Brasil & $\begin{array}{l}\text { Estudo } \\
\text { descritivo, } \\
\text { exploratório }\end{array}$ & 5 & $\mathrm{~B}$ \\
\hline $\mathrm{A} 2$ & $\begin{array}{l}\text { Perfil } \\
\text { adolescentes com } \\
\text { comportamentos de } \\
\text { autolesão } \\
\text { identificados nas } \\
\text { escolas estaduais } \\
\text { em Rolim de Moura- } \\
\text { RO. }\end{array}$ & $\begin{array}{l}\text { BIREME/ } \\
\text { BVS } 2017\end{array}$ & Brasil & $\begin{array}{l}\text { Estudo } \\
\text { retrospectivo }\end{array}$ & 5 & $A$ \\
\hline A3 & $\begin{array}{l}\text { Caracterização do } \\
\text { perfil } \\
\text { epidemiológico de } \\
\text { casos de suicídios } \\
\text { na cidade de Piripiri- } \\
\text { PI nos anos de } 2008 \\
\text { a } 2012 \text {. }\end{array}$ & $\begin{array}{l}\text { BIREME/ } \\
\text { BVS } 2017\end{array}$ & Brasil & $\begin{array}{l}\text { Estudo } \\
\text { descritivo, } \\
\text { exploratório }\end{array}$ & 5 & $A$ \\
\hline A4 & Vítimas do silêncio & $\begin{array}{l}\text { Pubmed/ } \\
2017\end{array}$ & Brasil & $\begin{array}{l}\text { Estudo } \\
\text { descritivo, } \\
\text { exploratório }\end{array}$ & 5 & $\mathrm{~B}$ \\
\hline A5 & $\begin{array}{lr}\text { Perfil } & \text { de } \\
\text { adolescentes } & \text { em } \\
\text { privação } & \text { de } \\
\text { liberdade: eventos } \\
\text { estressores, uso de } \\
\text { drogas } & \text { e } \\
\text { expectativas } & \text { de } \\
\text { futuro. } & \end{array}$ & PubMed/ 2014 & Brasil & $\begin{array}{l}\text { Estudo } \\
\text { transversal }\end{array}$ & 7 & $\mathrm{C}$ \\
\hline 6 & $\begin{array}{l}\text { Perfil de suicídios } \\
\text { em município da } \\
\text { Amazônia Legal. }\end{array}$ & EBSCO 2018 & Brasil & $\begin{array}{l}\text { Estudo } \\
\text { qualitativo }\end{array}$ & 5 & $A$ \\
\hline A7 & $\begin{array}{l}\text { ldeação suicida, } \\
\text { resolução } \\
\text { problemas, } \\
\text { expressão de raiva } \\
\text { e impulsividade em } \\
\text { dependentes de } \\
\text { substâncias } \\
\text { psicoativas. }\end{array}$ & EBSCO 2016 & Brasil & $\begin{array}{l}\text { Estudo } \\
\text { transversal }\end{array}$ & 7 & $B$ \\
\hline A8 & $\begin{array}{l}\text { Mal tratos físicos e } \\
\text { emocionais em } \\
\text { crianças e conduta } \\
\text { suicida no adulto. }\end{array}$ & EBSCO 2014 & México & $\begin{array}{l}\text { Estudo caso - } \\
\text { controle }\end{array}$ & 4 & $\mathrm{~B}$ \\
\hline
\end{tabular}

Fonte: BIREME, PUBMED e EBSCO, 2018. 
O quadro 3 evidenciou que a maioria das vítimas de tentativa de suicídio e de suicídio são do gênero masculino, com idade entre 10 a 40 anos. Quanto ao perfil emocional da pessoa suicida segundo os estudos, foi relacionado um perfil de pessoas com depressão, ansiedade, melancolismo, com baixo autoestima, sentindo-se desamparados, e com desesperança em relação a vida. Tal perfil revela que as vítimas de tentativa de suicídio já passavam por problemas emocionais antes de tentarem se suicidar, e que necessitavam de um acompanhamento profissional para minimizar esse quadro de desequilíbrio emocional.

Quadro 3 - Publicações incluídas que caracterizam o gênero e a idade amostral prevalente dos estudos, perfil emocional da pessoa suicida e meios utilizados para a tentativa de suicídio. Caxias, Maranhão, 2019.

\begin{tabular}{|c|c|c|c|c|}
\hline № de ordem & Autor/ano & Gênero & Idade & Perfil emocional da pessoa suicida \\
\hline $\bar{A} 1$ & $\begin{array}{l}\text { MOREIRA DL, } \\
\text { et al., } 2015 .\end{array}$ & Feminino & $\begin{array}{l}\text { Adultos } \\
\text { jovens com } \\
\text { até } 25 \text { anos. }\end{array}$ & $\begin{array}{l}\text { Pacientes melancólicos e depressivos } \\
\text { sem suporte psicológico ou } \\
\text { psiquiátrico. }\end{array}$ \\
\hline A2 & $\begin{array}{l}\text { SILVA MF, } \\
\text { SIQUEIRA AC, } \\
2017 .\end{array}$ & Masculino & $\begin{array}{l}\text { Adolescentes } \\
\text { de } 13 \text { a } 18 \\
\text { anos }\end{array}$ & $\begin{array}{l}\text { Adolescentes em conflito familiar, } \\
\text { sentindo-se desamparados } \\
\text { socialmente e melancólicos. }\end{array}$ \\
\hline A3 & $\begin{array}{l}\text { LEITE SCA, } \\
\text { FORTES AM, } \\
\text { VERAS CNSS, } \\
2017 .\end{array}$ & Masculino & $\begin{array}{l}\text { Jovens de } 10 \\
\text { a } 15 \text { anos. }\end{array}$ & $\begin{array}{l}\text { Pacientes relataram intenso } \\
\text { sofrimento emocional e sentimentos } \\
\text { de desesperança em relação a sua } \\
\text { vida. }\end{array}$ \\
\hline A4 & $\begin{array}{l}\text { BITTENCOURT } \\
\text { LABS, } \\
\text { ARRUDA E, } \\
2017 .\end{array}$ & Feminino & $\begin{array}{l}\text { Jovens entre } \\
15 \text { a } 30 \text { anos }\end{array}$ & $\begin{array}{l}\text { Desejo autodestrutivo, desesperança } \\
\text { e desespero psicológico. }\end{array}$ \\
\hline A5 & $\begin{array}{l}\text { NARDI FL, } \\
\text { JAHN GM, } \\
\text { DELL'AGLIO } \\
\text { DD } 2014 .\end{array}$ & Feminino & $\begin{array}{l}\text { Adolescentes } \\
\text { entre } 14 \text { a } 30 \\
\text { anos. }\end{array}$ & $\begin{array}{l}\text { Sentimentos de inutilidade, falta de } \\
\text { esperança, baixa autoestima. }\end{array}$ \\
\hline A6 & $\begin{array}{l}\text { SILVA E, } \\
\text { MARQUES } \\
\text { JUNIOR J, e } \\
\text { SUCHARA E, } \\
2018 .\end{array}$ & Masculino & $\begin{array}{l}\text { Adultos de } 20 \\
\text { a } 40 \text { anos. }\end{array}$ & $\begin{array}{l}\text { Angústia, } \\
\text { profunda. }\end{array}$ \\
\hline A7 & $\begin{array}{l}\text { ALMEIDA } \\
\text { RMM, et al., } \\
2016 \text {. }\end{array}$ & Masculino & $\begin{array}{l}\text { Adultos com } \\
\text { mais de } 25 \\
\text { anos }\end{array}$ & $\begin{array}{l}\text { Crise emocional, relatos de vontade de } \\
\text { sumir, desesperança, tristeza. }\end{array}$ \\
\hline A8 & $\begin{array}{l}\text { KOKOULINA E, } \\
\text { FERNÁNDEZ } \\
\text { R, } 2014 \text {. }\end{array}$ & Masculino & $\begin{array}{l}\text { Adolescentes } \\
\text { entre } 12 \text { a } 20 \\
\text { anos. }\end{array}$ & $\begin{array}{l}\text { Depressão, } \\
\text { autoestima. }\end{array}$ \\
\hline
\end{tabular}

Fonte: BIREME, PUBMED e EBSCO, 2019.

\section{DISCUSSÃO}

Através dessa revisão integrativa foi possível evidências que a faixa etária de 10 a 40 anos, sendo constituída em sua maioria por jovens, do gênero masculino, e com desequilíbrio emocional foram os principais fatores de risco para o suicido. Sobre esse fato Nunes $F(2016)$ relatou que os jovens passam por uma mudança muito forte de fase da vida, deixam de assumir o caráter infantil para lidar com o cotidiano difícil de um adulto que tem inúmeras responsabilidades sociais e financeiras, que podem ser mal interpretadas causando estresse, angustia e depressão, tais fatores podem servir como gatilho para a ideação suicida.

Freitas G (2015) revelou ainda que os jovens, principalmente entre 15 a 25 anos enfrentam dilemas próprios relacionados ao amadurecimento e ao futuro, tais fatores e o desamparo social e familiar funcionam como gatilho para o agravamento de problemas emocionais, além disso, os jovens são mais expostos ao uso de substancias como álcool e outras drogas que agem deprimindo o sistema nervoso. 
Silva MF e Siqueira AC (2017) esboçaram que o fator masculinidade reprime o lado emocional do homem, podendo gerar uma sobrecarga psicológica desencadeante de pensamentos suicidas, além de que culturalmente o homem é o encarregado pelo sustento de sua família, e fatores financeiros tem desencadeado uma ampla taxa de depressão e consequente tentativas de suicídio.

Nos artigos selecionados para a construção dessa revisão integrativa, foi possível verificar que, o perfil das pessoas suicidas demonstra muitos desequilíbrios emocionais, tais como ansiedade, depressão, baixa autoestima, melancolia, desamparo, desesperança e outros sentimentos depreciativos. Tais dados corroboram com o estudo de Heck RM, et al. (2012) onde foi evidenciado também tais características de desequilíbrio emocional entre os participantes do estudo do referido autor.

Segundo Gonçalves RC (2011) os suicidas têm perfil emocional desestabilizado, geralmente produzem sentimentos de forma elevada, e se sentem reprimidos ou hostilizados por atitudes simples. Os autores informaram ainda que essas pessoas enxergam o suicídio como uma formal viável de alívio para sua intensa desestabilidade emocional.

Oliveira SR (2012) informou ainda que a vulnerabilidade psicológica, como por exemplo, pessoas que sofrem de depressão ou bipolaridade, combinados com uma falta de apoio profissional geralmente acarreta em várias tentativas de suicídio. As maiorias dos suicidas relatam ou deixam pistas aos familiares e amigos do tanto que estão sofrendo emocionalmente, porem existem suicidas que escondem seu desejo de morte e não deixam transparecer o seu adoecimento emocional.

Nos resultados dessa revisão integrativa, foi verificado é necessário o estabelecimento de algumas medidas preventivas ao suicídio, como acompanhamento profissional, inclusão em terapias sejam elas individuais ou coletivas, uso de fármacos prescritos por médico psiquiatra e inclusão social através de grupos de apoio ou exercícios em grupo. Sobre essa ótica Almeida RMM, et al. (2013) explanaram que os profissionais de saúde ou até mesmo pessoas próximas ao paciente devem ficar atentos a sinais de alerta como relatos de infelicidade, distanciamento social, automutilação, perda de entes queridos ou demissão, problemas financeiros que afetam o emocional de pessoa e outras ocasiões que causem grande desordem emocional, como por exemplo, um rompimento afetivo.

Freitas G (2015) ainda evidência sobre os sinais de alerta para casos de tentativas anteriores de suicídio, transtorno mental, doenças graves, ansiedade e desesperança, crise conjugal e familiar, situações de luto ou perda, facilidade de acesso aos meios de interrupção da vida, e a influência de outras pessoas para que a vítima se mate.

Conforme o enunciado de Nunes $\mathrm{F}$ (2016), a equipe assistencial deve avaliar o estado mental das pessoas que deseja se suicidar, avaliar o motivo dela querer encerrar sua vida e os planos que possui de como fazer isso, para tal é necessário criar um vínculo de confiança com o paciente para entender seus planos suicidas e elaborar estratégias eficaz de prevenção de futuras tentativas de suicídio.

Moreira DL, et al. (2015) evidenciaram ainda que é necessário trabalhar sobre os sentimentos suicidas do paciente, uma vez que quanto mais abertamente a pessoa fala sobre os fatores que o motivam tentar se matar, menos turbulentas suas emoções se tornam, desse modo quando a turbulência emocional cede, a pessoa pode se tornar reflexiva, e enxergar soluções para seus problemas que não sejam a morte, ou ainda sentirem-se valorizadas por ter pessoas que se importam com suas queixas.

Segundo Heck RM, et al. (2012), os profissionais de saúde possuem importante papel na prevenção do suicídio, pois além deles garantirem uma assistência de qualidade e de acordo com as necessidades físicas e psicoemocionais dos pacientes, eles servem como apoio para a saúde mental dessas pessoas se restabelecerem, e consequentemente contribuem para a diminuição das taxas de suicido.

Freitas G (2015) explanou que os pacientes com propensão ao suicídio que são acompanhados por profissionais de saúde, sejam eles psicólogos, psiquiatras ou enfermeiros, têm um melhor enfrentamento dessa patologia, pois conseguem seguir as propostas terapêuticas de modo mais eficaz quando possui alguém habilitado para prestar-Ihes os cuidados adequados, como horário certo de medicações, inserção e 
encorajamento de seguir nas terapias, aconselhamento e escuta dos seus anseios para melhor tratar sua condição psicológica.

Para Oliveira SR (2012) a relevância dos profissionais de saúde na prevenção do suicídio é ainda maior na população de 13 a 19 anos, onde cerca de 34\% dos casos podem ser prevenidos quando o jovem busca aconselhamento com um profissional de saúde, visto que essa fase é naturalmente marcada por uma alteração de sentimentos e mudanças físicas. Nesse sentido a terapia e o aconselhamento profissional podem auxiliar os jovens suicidas a atravessaram por seus medos e anseios, e através de técnicas de redução de ansiedade e uso de antidepressivos prescritos, os pacientes podem enxergar um novo horizonte para a sua vida.

Ainda segundo a perspectiva anterior, Nunes $F$ (2016) esboçou que os profissionais de saúde auxiliam a população conscientizando-os sobrea importância do acompanhamento psicológico ou psiquiátrico, e dão maior ênfase para os sintomas de alterações emocionais e psicológicas para que os familiares ou responsáveis pelos pacientes o ajudem e os encorajem a procurar ajuda profissional, uma vez que as desordens emocionais são a base para a intenção suicida.

\section{CONSIDERAÇÕES FINAIS}

Através dessa revisão integrativa de literatura pode-se concluir que os jovens, do gênero masculino e com desordens emocionais são as pessoas mais propensas a tentarem praticar o suicídio. Os profissionais de saúde têm importante papel na prevenção do suicídio, pois eles garantem uma assistência de qualidade e de acordo com as necessidades físicas e psicoemocionais dos pacientes. Tendo em vista que o perfil de jovens com desequilíbrios emocionais estão mais associados às tentativas de suicídio, este estudo teve pilar de investigação voltado para assistência da equipe de saúde da pessoa jovem com a finalidade de alertar sobre o aumento das taxas de óbitos por suicídio devido a ausência ou precariedade da atenção à saúde mental.

\section{REFERÊNCIAS}

1. ALMEIDA RMM, et al. Ideação suicida, resolução de problemas, expressão de raiva e impulsividade em dependentes de substâncias psicoativas. Revista Brasileira de Enfermagem, 2013; 3(7): 67-81.

2. BITTENCOURT LABS, ARRUDA E. Vítimas do Silêncio. Revista São Luis Orione, 2017; 1(11): 1-14.

3. DUTRA EMS. Depressão e suicídio em crianças e adolescentes. Revista Mudanças, 2008; 9(15): 27-35.

4. FREITAS G. A morte pode esperar? Clínica psicanalítica do suicídio. Stylus Revista de psicanálise, 2015; 31(7): 215222.

5. GONÇALVES RC, GONÇALVES E, OLIVEIRA JÚNIOR LB. Determinantes espaciais e socioeconômicos do suicídio no Brasil: uma abordagem regional. Revista Nova Economia, 2011; 21(2): 281-316.

6. HECK RM, et al. Ação dos profissionais de um Centro de Atenção psicossocial diante de usuários com tentativa e risco de suicídio. Revista Texto Contexto Enfermagem, 2012; 21(1): 26-33.

7. KOKOULINA E, FERNÁNDEZ R. Maltrato físico y emocional en la infancia y conducta suicida en el adulto. Revista de Psicopatología y Psicología Clínica, 2014; 19(2): 93-103.

8. LEITE SCA, FORTES AM, VERAS CNSS. Caracterização do perfil epidemiológico de casos de suicídios na cidade de Piripiri-PI nos anos de 2008 a 2012. Revista Interdisciplinar, 2017; 10(1): 103-109.

9. MOREIRA DL et al. Perfil de pacientes atendidos por tentativa de suicídio em um centro de assistência toxicológica. Revista Observatório do Cuidado, 2015; 14(6): 27-20.

10. NARDI FL, JAHN GM, DELL'AGLIO DD. Perfil de adolescentes em privação de liberdade: eventos estressores, uso de drogas e expectativas de futuro. Psicologia em Revista, 2014; 20(1): 116-137.

11. NUNES F. O fenômeno do suicídio entres os familiares sobreviventes: revisão integrativa. Revista Portuguesa de Enfermagem de Saúde Mental, 2016; 15(7): 145-159.

12. OLIVEIRA SR. Sobre suicídio na Clínica Junqueira com pacientes adolescentes. O mundo da Saúde, 2012; 36(1): 103-110.

13. SILVA E, MARQUES JJ, SUCHARA E. Perfil de suicídios em município da Amazônia Legal. Cadernos Saúde Coletiva, 2018; 26(1): 34-47.

14. SILVA MF, SIQUEIRA AC. O perfil de adolescentes com comportamentos de autolesão identificados nas escolas estaduais em Rolim de Moura-RO. Revista Farol, 2017; 3(3): 5-20. 